\title{
Simultaneously Performed, Totally Endoscopic Left Atrial Myxoma Resection and Lobectomy
}

\author{
Jin Shi, MM, ${ }^{1,2}$ Yong Wang, MM, ${ }^{2}$ Qiuyun Wang, MM, ${ }^{3}$ Xiaohan Bing, MM, ${ }^{1}$ Zengshan Ma, MD $^{1}$ \\ ${ }^{1}$ Department of Cardiac Surgery, Qilu Hospital, Shandong University, Jinan, China; ${ }^{2}$ Department of Cardiac Surgery, Linyi People's \\ Hospital, Linyi, China; ${ }^{3}$ Department of Cardiac Surgery, Chinese Medicine Hospital in Linyi City, Linyi, China
}

\section{ABSTRACT}

The patient was a 69 -year-old male patient with cancer in the right lung and whose preoperative examination showed left atrial myxoma. Simultaneous surgery for both cardiac myxoma resection and a lobectomy by totally endoscopic surgery without robotic assistance was performed. First, the cardiac tumor on the heart was removed using a cardiopulmonary bypass (CPB), then a lobectomy without any new incisions was performed. This case provides evidence that in individual select patients, a left atrial myxoma resection and lobectomy can be performed under total endoscopy at the same time.

\section{INTRODUCTION}

Cardiac atrial myxomas represent approximately 50\% of all cardiac tumors. Most benign cardiac myxomas originate from a specific location in the left atrium. Some malignant cardiac myxomas have multiple origins and family aggregation [Saad 2018]. As cardiac myxomas may lead to systemic thromboembolic events from tumor fragmentation, almost all of them require surgery [Ali 2018]. Pulmonary nodules are common in clinical practice, and they usually require surgical treatment. Nevertheless, the simultaneous occurrence of a left atrial myxoma and lung cancer is quite rare. Based on previous experience, patients with two non-related diseases tend to choose staged surgery with the condition considered most life-threatening being given surgical priority. Based on this previous experience, operating is the conventional practice, and we would perform the removal of the atrial myxoma first, and then perform pulmonary surgery. With staged surgery, there often can be surgical complications, and these can increase the difficulty of the operation [Yang 2016]. A combined surgical procedure can be accepted when patients are assessed for their tolerance to surgery. The best option is to perform a left atrial myxoma resection and lobectomy using endoscopic surgery without robotic assistance, of which there is no literature yet reported. Here, we

Received December 11, 2019; received in revised form April 15, 2020; accepted April 22, 2020.

Correspondence: Zengshan Ma, Department of Cardiac Surgery, Qilu Hospital, Shandong University, Finan, China; (e-mail: mazengshansdu@gmail.com). describe a case of simultaneous surgery for both cardiac myxoma resection and lobectomy using endoscopic surgery without robotic assistance.

\section{CASE REPORT}

A 69-year-old man was diagnosed with lung cancer, during a routine medical examination that included a CT scan of the chest area. He showed no apparent signs or symptoms. Enhanced chest CT showed a lung nodule $(2.9 \times 2.6 \mathrm{~cm})$ with thin, hairy stabs located at the right upper lobe (Figure 1).

Positron emission tomography (PET) was used to measure glucose metabolism (18F-FDG), which showed high uptake of FDG in the posterior segment of the right upper lobe, approximately $3.2 \times 2.8 \mathrm{~cm}$, SUV max 18.5 , SUV mean 11.8 , and no apparent abnormalities in any other part of the body. The preoperative echocardiography (ECG) examination showed that the patient had a left atrial myxoma with an oval-shaped tumor in the left atrium $(2.4 \times 3.4 \mathrm{~cm})$ adhering to the atrial septal; otherwise, the pathology of the valve and the heart function was normal. The pulmonary function test showed the patient with mild obstructive ventilation dysfunction before surgery. The patient did not have other underlying diseases. Patients can tolerate simultaneous double surgery. We first performed a cardiac myxoma resection because it is a Type I incision operation, whereas a lobectomy is Type II incision surgery. Moreover, the risk from anesthesia is reduced if we perform the operations in this order.

The patient was placed in a supine position with the right side of their body raised approximately $10-15^{\circ}$, and the right shoulder extended. External defibrillator patches were attached. General anesthesia and double cavity intubation were used during the operation. Femoral arterial and venous cannulation (Medtronic, Inc, Minneapolis, Minn.) were performed using the Seldinger guidewire technique to establish $\mathrm{CPB}$, and an internal jugular venous (IJV) catheter also was used. Three $10 \mathrm{~mm}-15 \mathrm{~mm}$ thoracic incisions were made. The first incision for the left-hand operation was located in the right parasternal line third intercostal space (ICS); the second incision for right-hand operation was located in the outside of the right clavicle midline fifth ICS; the third incision for thoracoscopy was found in the fourth ICS anterior axillary line [Ma 2019]. The catheter of the left heart through the right superior pulmonary vein was inserted, and carbon dioxide continuously was injected into the thoracic cavity during the 


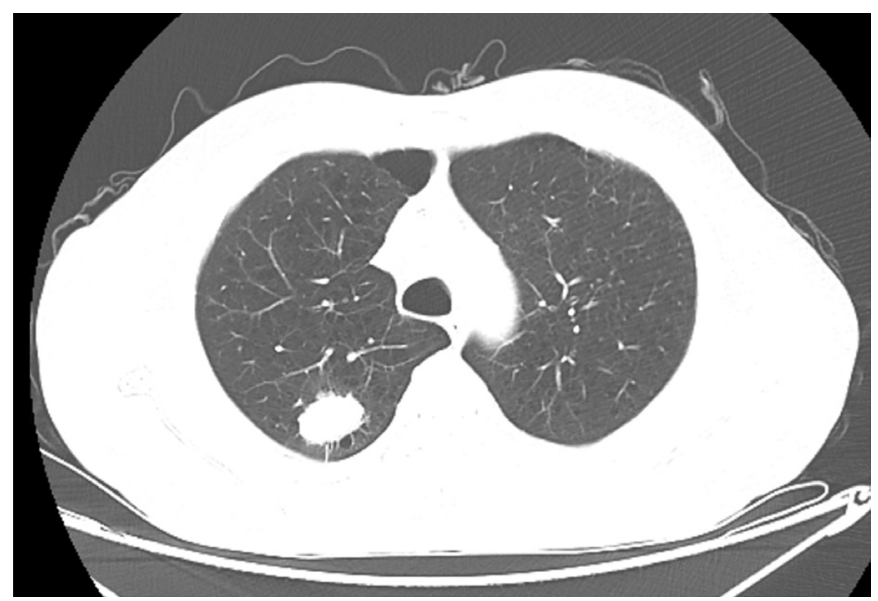

Figure 1. CT scans showed lung nodule located at the right upper lobe.

operation. We made an incision through the interatrial sulcus, and the excision of the entire mass was achieved (Figure 2).

Following the myxoma resection operation, the incisions were covered with a sterile dressing, and the patient was moved into a left lateral decubitus position. We sterilized the operative field once more, and then a right upper lobe resection and lymphadenectomy in the hilar and mediastinum were performed through primary incisions. The tumor was located at the right upper lobe near the pulmonary hilar and reached visceral pleural. The patient underwent a right upper lobectomy and mediastinal lymph node resection. The entire operation process took approximately 4.5 hours. Pathology and immunohistochemistry displayed benign cardiac myxoma (elastic fiber staining+, PAS+, PAS-D+, ) and lung adenocarcinoma (TTF-1+, CK7+, CK5/6+, P63-, P40-, Syn-, CgA-, CD56-, Napsin-A+, CK20-, CD31+, D2-40+, Ki-67+). 2018 new TNM classification: T1cN0M0, Stage IA3. The patient suffered a small amount of pleural effusion and was discharged 18 days after the operation.

\section{DISCuSSION}

Video-assisted thoracoscopic surgery (VATS) is widely used in thoracic surgery. Still, in cardiac surgery, endoscopic operation is not yet widely used, particularly totally endoscopic heart operations without any robotic assistance. Minimally invasive surgery gives less trauma to the patients. The endoscopic procedure provides us with a novel, minimally invasive alternative to perform a left atrial myxoma resection and right lobectomy simultaneously through the right thoracic cavity. Of course, when the patient suffers from atrial myxoma and left lung disease, incisions must be made on the bilateral thoracic cavity. We must neutralize the heparin following the heart operation to reduce bleeding during lung surgery.

It should be noted that late complications of extracorporeal circulation contribute indirectly to the expansion of the existing malignancy, according to the international bibliography. It also is suggested that the $\mathrm{CPB}$ machine, as well as the cell saver devices, can safely be used with patients who

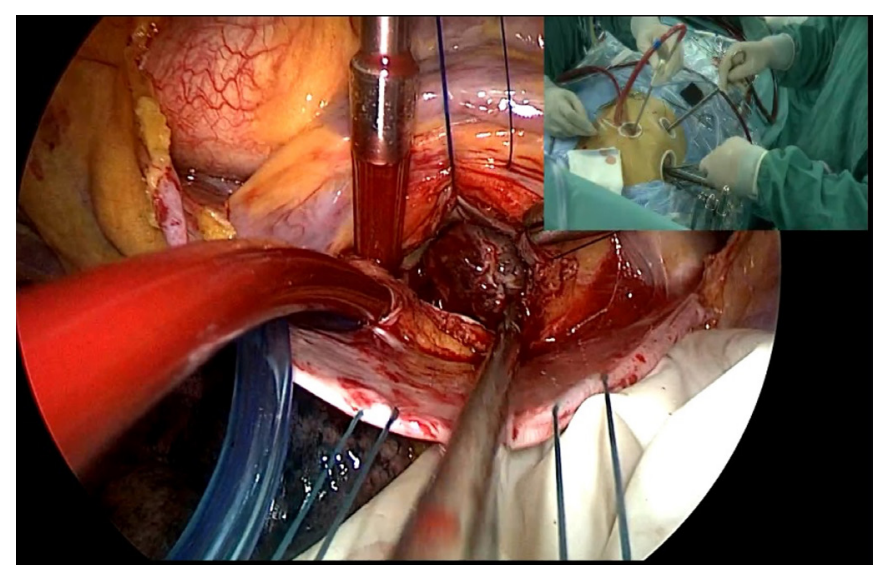

Figure 2. Completely removing the left atrial myxoma.

have malignant disease without the increase of risk of hematogenous dissemination in cases of malignancy coexistence [Reynen 1995]. The long-term survival of patients reported in the literature mainly is related to tumor staging, the incidence of cardiac accidents is very low, and the metastatic spread of tumors has a significant impact on prognosis. There are no guidelines for the treatment of the multi pathology. Other cardiac surgeries, such as valve replacement with $\mathrm{CPB}$ and colectomy, also have been reported [Choi 2009]. Before we excise the myxoma with the heart beating, we make sure there is an appropriate flow of left atrium cannulation and venous cannulation for the operative field exposure and use carbon dioxide during CPB to avoid air embolism. There does not necessarily have to be cardiac arrest or blocking of the ascending aorta during the operation, and this can lower the inflammatory reaction and myocardial injury, and thereby lower the possibility of tumor diffusion [Ma 2014]. The lung nodule was close to the hilum, so we performed a complete lobectomy instead of a wedge resection. Cardiac surgery generally should be performed first in patients who have surgical indications for both cardiac surgery and a malignant neoplasm. Some scholars believe that the operative trauma during the same period is too significant, the operation and anesthesia time is extended, hemostasis is difficult, and risk of postoperative bleeding is high. Medical costs also are higher. The most important thing is that if the heart surgery delays the tumor removal, tumor progression and distant metastasis may occur; if the lung tumor is removed first, the incidence of perioperative cardiovascular accidents significantly increases. For the above reasons, we chose a single period of surgery. If the patient decides staggered surgery, whether it is a minimally invasive resection of a heart tumor or lung tumor, the patient usually needs to choose a thoracotomy when the other operation is performed because the adhesion of the chest cavity will make the endoscopic surgery difficult to carry out and result in the patient losing the chance of minimally invasive surgery, thereby increasing surgical trauma. But in cases like this, we may have a new choice: To perform simultaneous operations on the pulmonary nodules and atrial myxomas using thoracoscopic techniques. 


\section{CONCLUSION}

For patients who are suffering from lung nodules and heart disease, apart from staged surgery and median thoracotomy, totally endoscopic surgery without robotic assistance offers a new and valid solution. A cardiac myxoma resection provides a much safer surgical environment for a lobectomy, and the lobectomy can be performed without making any new incisions. This simultaneous surgery also can avoid thoracic adhesion and reduce any difficulties of staging surgery.

\section{REFERENCES}

Ali MU, Finkel J. Atrial Myxoma. 2018. N. Engl. J. Med 379(16):e26.
Choi PW, Kim CN, Chang SH,et al. 2009. Cardiac metastasis from colorectal cancer: a case report. World J astroenterol 15(21):2675-8.

Ma ZS. 2019. Clinical Application of Total Thoracoscopic Technique in Cardiac Surgery. People's Medical Publishing House 11-38, 83-85.

Ma ZS, Yang CY. 2014. Totally thoracoscopic closure of ventricular septal defect without a robotically assisted surgical system: a summary of 119 cases. J Thorac Cardiovasc Surg 147(3):863-8.

Reynen K. Cardiac myxomas. 1995. N Engl J Med 333: 1610-1617.

Saad AM, Abushouk AI, Al-Husseini MJ, et al. 2018. Characteristics, Survival and Incidence Rates and Trends of Primary Cardiac Malignancies in the United States. Cardiovasc Pathol 33:27-31.

Yang Y, Xiao F, Wang J, et al. 2016. Simultaneous surgery in patients with both cardiac and noncardiac diseases. Patient Prefer Adherence 10: 1251-1258. 